\title{
Luftverbesserung lässt Kinder aufatmen
}

\author{
Verbesserungen der Luftqualität, vor allem hinsichtlich Feinstaub und Stickstoffdioxid, gehen langfristig \\ mit einer Verbesserung der Lungenfunktion und geringeren Raten an Asthma bei Kindern einher.
}

_ In Los Angeles wurden drei Kohorten der Geburtsjahrgänge 1994-1998, 19972001 und 2007-2011 mittels Lungenfunktion untersucht. Das mediane Alter der insgesamt 2.120 Kinder lag zu Beginn der Untersuchung bei elf Jahren, am Ende bei 15 Jahren. Per linearer Regression wurden Korrelationen zwischen der Luftverschmutzung und der Einsekundenkapazität $\left(\mathrm{FEV}_{1}\right)$ sowie der forcierten exspiratorischen Vitalkapazität (FVC) gebildet. Die Eltern füllten regelmäßig Fragebögen zu Gesundheitszustand, pulmonalen Symptomen, Exposition gegenüber Zigarettenrauch und sozialen Umständen aus.
Über 13 Jahre hinweg ergab sich eine klare Korrelation zwischen der Zunahme von $\mathrm{FEV}_{1}$ und FVC mit abnehmenden Luftkonzentrationen von Stickstoffdioxid und Feinstaub, sowohl für Teilchen $<2,5 \mu \mathrm{m}$ als auch $<10 \mu \mathrm{m}$. Die Assoziationen blieben auch nach Korrektur für potenziell konfundierende Faktoren erhalten und waren bei Kindern mit und ohne Asthma feststellbar. In der ersten Kohorte hatten 7,9\% der Kinder im Alter von 15 Jahren eine $\mathrm{FEV}_{1}$ unter $80 \%$ des Vorhersagewerts. In den späteren Kohorten waren es 6,3\% bzw. 3,6\%.

- Gauderman WJ et al. Association of improved air quality with lung development in children. NEngl J Med. 2015;372:905-13

\section{KOMMENTAR}

Der Zusammenhang scheint auch für Laien nachvollziehbar zu sein, doch ist es mit Logik und gesundem Menschenverstand nicht immer getan, wenn es um den Abschied von bequemen Gewohnheiten und erhebliche politische und ökonomische Umwälzungen geht. Daher braucht es Wissenschaft. Zum Glück sprechen die Daten auch dieser Untersuchung eine so klare Sprache, dass auch die Lobby der Auto- und Erdölindustrie ihre Positionen nicht halten können wird - in der Tat sehen wir hier ja seit einiger Zeit Bewegung. Und das, obwohl Assoziationen eigentlich keine Kausalität beweisen.

Prof. Dr. med. H. S. FüeßI

\section{Aus diesem Kerker kommt die Lunge allein nicht heraus}

Eine 46-jährige Frau mit fortgeschrittener Leberzirrhose litt unter rezidivierender Atemnot aufgrund eines hepatischen Pleuraergusses rechts, der zahlreiche Pleurapunktionen erforderlich machte. Das Atemgeräusch auf der rechten Thoraxhälfte war völlig aufgehoben. Radiologisch zeigte sich ein Hydropneumothorax (Abb. A). Der Pleuraerguss wurde mithilfe eines Pigtail-Katheters dauerhaft drainiert. Allerdings verblieb der Pneumothorax (Abb. B). In der CT wurde klar, warum sich die Lunge nicht entfaltete. Der gesamte rechte Flügel war durch einen chronischen entzündlichen Prozess narbig verdickt, was eine Entfaltung verhinderte (Abb. C). Der so entstehende große Pleuraraum wurde durch Flüssigkeit ausgefüllt.
Lässt man in einem solchen Fall die Flüssigkeit ab, entsteht ein Pneumothorax ex vacuo, da die gefangene Lunge die Thoraxhöhle nicht ausfüllen kann. Nach Ausschluss anderer Ursachen, etwa einer endobronchialen Obstruktion, muss man die verdickte viszerale Pleura chirurgisch entfernen, um der Lunge die Expansion zu ermöglichen. Diese Operation erfolgte bei der Patientin im Rahmen eines thorakoskopischen Eingriffs mit Dekortikation und Pleurodese. Die rechte Lunge entfaltete sich anschließend vollständig.

Prof. Dr. med. H. S. FüeßI

- Albores J, Wang T (Korres.jeffalbores@yahoo.com): Trapped lung. NEnglJMed. 2015;372:e25
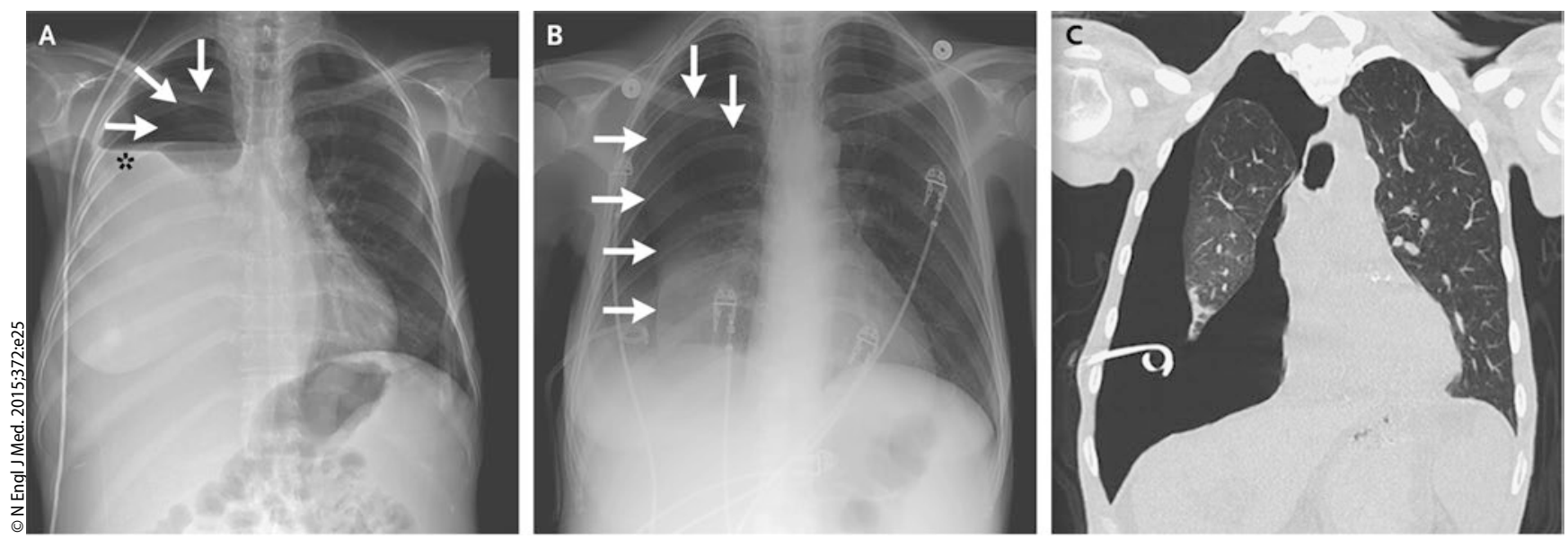

A: Hydropneumothorax im Röntgenbild (Pfeile: Pneumothorax, Sternchen: Flüssigkeitsspiegel). B: Pneumothorax nach Drainage des Ergusses (Pfeile). C: Thorax-CT mit narbig verdickter rechter Lunge. 